\title{
Prognosis of young patients with monoclonal gammopathy of undetermined significance (MGUS)
}

\author{
Li Pang $\mathbb{1}^{1}$, S. Vincent Rajkumar $\mathbb{E}^{2}$, Prashant Kapoor ${ }^{2}$, Francis Buadi², Angela Dispenzieri $\mathbb{1}^{2}$, Morie Gertz $\mathbb{0}^{2}$, \\ Martha Lacy ${ }^{2}$, Robert Kyle ${ }^{2}$ and Shaji Kumar $\mathbb{B}^{2}$
}

\begin{abstract}
Monoclonal gammopathy of undetermined significance (MGUS) is rare in young patients (age $<40$ years at diagnosis), with a prevalence of $<0.3 \%$, representing $\sim 2 \%$ of all patients with MGUS. We hypothesized that MGUS detected in young patients may be associated with a higher risk of progression. We examined 249 patients with MGUS < 40 years old. Among these, 135 patients had immune-related conditions, including infections, autoimmune and inflammatory disorders at the time of diagnosis of MGUS. The risk of progression to multiple myeloma or a related disorder at 5 years and 10 years was $6.0 \%$ and $13.8 \%$, respectively. The size of $\mathrm{M}$ protein was a significant risk factor for progression (HR $4.2,95 \% \mathrm{Cl} 2.2-7.9)$. There was a trend that the risk of progression was higher in patients without immune-related conditions (HR 2.36, 95\% Cl 0.85-6.52, $p=0.088$ ). The M protein resolved in $36(14 \%)$ patients, with a greater likelihood of resolution in patients with immune-related conditions (RR 1.9, 95\% Cl 1.02-3.6). Young patients with MGUS have a similar risk of progression as older patients, 1.4\% per year. Over 50\% are diagnosed in the setting of immune-related disorders. Patients with immune-related disorders may have a lower risk of progression.
\end{abstract}

\section{Introduction}

Monoclonal gammopathy of undetermined significance (MGUS) is defined as the presence of monoclonal $(\mathrm{M})$ protein $\leq 3 \mathrm{gm} / \mathrm{dl}$ in the peripheral blood, plasma cells $<10 \%$ in the bone marrow and the absence of end organ damage or CRAB features that are related to $M$ protein or clonal plasma cells. MGUS is perceived as a premalignant entity of multiple myeloma and lymphoplasmacytic malignancies in patients older than 50 years ${ }^{1}$. The progression rate of MGUS in these patients to malignant disorders is about $1 \%$ per year ${ }^{2}$. The prevalence of MGUS in young patients (age < 40 years) is $<0.3 \%$, representing about $2 \%$ of all patients with

\footnotetext{
Correspondence: Shaji Kumar (kumar.shaji@mayo.edu)

${ }^{1}$ Department of Medicine, NYC Health + Hospitals/Jacobi, Albert Einstein College of Medicine, Bronx, NY, USA

2Division of Hematology, Department of Internal Medicine, Mayo Clinic, Rochester, MN, USA
}

MGUS $^{1,3,4}$. The clinical course of young MGUS patients remains largely unknown.

A variety of risk factors have been associated with risk of progression from MGUS to other plasma cell disorders, but their impact has not been specifically evaluated in the younger patients with MGUS. Specifically, the impact of immune-related conditions, including autoimmune, infectious and inflammatory disorders occurring in the context of monoclonal gammopathy has not been studied. Lindqvist et al reported in a large case-control study that the risk of MGUS was higher in patients with all categories of immune-related conditions ${ }^{5}$. Similar relations were highlighted on multiple myeloma (MM) by Brown et al. that patients with immune-related conditions were $15-29 \%$ more likely to develop $\mathrm{MM}^{6}$. Autoimmune diseases have been associated with increase in the risk of MGUS and $\mathrm{MM}^{7}$. In the patients with autoimmune diseases, the age of onset of MM is earlier ${ }^{8,9}$. Constant stimulation to immune system in the immune-related 
conditions can cause B cell dysfunction and has been hypothesized as a risk for development of clonal plasma cell disorders ${ }^{10,11}$.

We designed the current study to better evaluate the natural history of MGUS in young patients and to specifically examine if there is a more aggressive clinical course in terms of disease progression to $\mathrm{MM}$ or a related disorder. Our study examined the prognosis of MGUS in a large number of young MGUS patients, and in particular the impact of concurrent immune-related conditions on the progression of MGUS.

\section{Patients and methods}

From a prospectively maintained Dysproteinemia database at Mayo Clinic, Rochester, we identified 249 patients who were younger than 40 years at the time of diagnosis of MGUS and evaluated between January 1, 1997 and December 31, 2016. All patients met the criteria of MGUS and had a serum $M$ protein no greater than $3 \mathrm{~g} / \mathrm{dl}$ and $10 \%$ or fewer bone marrow plasma cells if a bone marrow evaluation was performed. Exclusion criteria included patients with MGUS who presented with peripheral neuropathy and subsequently diagnosed as POEMS syndrome, and those who had prior lymphoid malignancies and received chemotherapy or prolonged ( $>4$ weeks) steroids prior to MGUS diagnosis. Patients with biclonal gammopathy were analyzed using the larger $M$ protein. Patients with light-chain MGUS were not included in analysis involving $M$ protein. The Mayo Clinic Institutional Review Board approved the study and it was conducted in accordance with the Declaration of Helsinki and the Health Insurance Portability and Accountability Act guidelines of 1996.

We obtained information of all patients included on age, sex, chief complaints closest to the date of MGUS diagnosis, and family history of any plasma cell disorders or hematologic malignancy. Monoclonal protein isotypes and the size of $\mathrm{M}$ protein were obtained from the day of MGUS diagnosis. The results of beta2 microglobulin, lactate dehydrogenase, serum free light chain ratio, bone marrow plasma cell percentage (if available) were used if the tests were done at least 3 months before progression. When $\mathrm{M}$ protein was detected only by immunofixation but was too small to be quantifiable on electrophoresis, the size of $\mathrm{M}$ protein was coded as $0.001 \mathrm{~g} / \mathrm{dl}$. Data on immune-related conditions were collected from the inpatient and outpatient records which included autoimmune, inflammatory, and infectious diseases. Patients were segregated into two groups according to the presence or absence of any of the immune-related conditions at the time of MGUS diagnosis.

The endpoint of our study was progression from MGUS to smoldering multiple myeloma (SMM), MM, macroglobulinemia, non-Hodgkin lymphoma, immunoglobulin (heavy) light chain amyloidosis or chronic lymphocytic leukemia. The patients were found to have SMM when their clinical presentations raised suspicion for disease progression and warranted further work up such as bone marrow biopsy, however, the concerning clinical symptoms were not from myeloma-defining events and the laboratory parameters did not fulfill a diagnosis of active MM. We diagnosed SMM when it met the diagnostic criteria of $\mathrm{M}$ spike $>3 \mathrm{~g} / \mathrm{dl}$ and/or $10-59 \%$ bone marrow clonal plasma cell during the follow up plus absence of myeloma-defining events. Most patients (67\%) did not have bone marrow biopsy when MGUS was diagnosed consistent with our clinical practice. None of the patients in the current study received treatment at the SMM stage. We did not observe a progression to active MM in this cohort. The occurrence and date of $\mathrm{M}$ protein resolution were documented when immunofixation tests were negative on two consecutive assessments at least 6 months apart during the follow-up.

Chi square and Wilcoxon rank-sum tests were applied to assess the statistical significance of differences in the basic features between the group of patients with immune-related conditions and the group of patients without immune-related conditions. Progression-free survival (PFS) and overall survival (OS) for the whole cohort and between the two groups were assessed by Kaplan-Meier method, using Wilcoxon and Log-rank tests for comparison. Age, size of $M$ protein, type of immunoglobulin, type of light chain and the presence of immune-related conditions were assessed in the Cox univariate progression model. The Cox multiparameter progression model was applied to assess the independent prognostic impact on PFS including age, the size of $M$ protein and the presence of immune-related conditions. The above methods to analyze PFS and OS were reapplied when the patients in whom the $\mathrm{M}$ protein disappeared were excluded. A two-tailed $p$ value $<0.05$ was considered significant for all statistical tests. JMP ${ }^{\circledR}$ Pro 14.0 (SAS Institute Inc., Cary, NC, USA) was used for statistical analysis.

\section{Results}

We identified 249 patients younger than 40 years at the time of diagnosis of MGUS who met the inclusion criteria. Their baseline demographic and laboratory parameters at the time of MGUS diagnosis are shown in Table 1 . The median age was 35 years; $130(52 \%)$ were women. Most of the patients $(n=179,72 \%)$ were diagnosed between the age of 30-39 years. A concurrent immune-related condition was present at the time of MGUS diagnosis in 135 (54\%) patients. The immunerelated conditions are shown in Table 2. A family history of hematologic malignancy was present in 14 (6\%) patients, including $6(2 \%)$ patients with plasma cell 
Table 1 Characteristics of the patients.

\begin{tabular}{|c|c|c|c|c|c|c|c|}
\hline Characteristics & $\begin{array}{l}\text { Entire cohort } \\
(N=249)\end{array}$ & & $\begin{array}{l}\text { Patients without } \\
\text { immune-related } \\
\text { conditions } \\
(N=114)\end{array}$ & & $\begin{array}{l}\text { Patients with immune- } \\
\text { related conditions } \\
(N=135)\end{array}$ & & $P$ value \\
\hline Female Sex (\%) & $52 \%$ & & $58 \%$ & & $47 \%$ & & 0.0988 \\
\hline $\begin{array}{l}\text { Age at MGUS diagnosis } \\
\text { median (yr) }\end{array}$ & 35 & $10-39$ & 35 & $12-39$ & 34 & $10-39$ & 0.0652 \\
\hline $\begin{array}{l}\text { Size of M protein } \\
\text { mean, IQR1-3 (g/dL) }\end{array}$ & 0.27 & $0.001-0.43$ & 0.36 & $0.001-0.60$ & 0.20 & $0.001-0.30$ & 0.0572 \\
\hline Subtypes of immunoglobulins & & & & & & & 0.2762 \\
\hline $\operatorname{lgG}($ no., \%) & 173 & $69.48 \%$ & 82 & $71.93 \%$ & 91 & $67.41 \%$ & \\
\hline $\lg \mathrm{A}($ no., \%) & 25 & $10.04 \%$ & 13 & $11.40 \%$ & 12 & $8.89 \%$ & \\
\hline $\lg M(n o ., \%)$ & 38 & $15.26 \%$ & 15 & $13.16 \%$ & 23 & $17.04 \%$ & \\
\hline light chain only (no., \%) & 1 & $0.40 \%$ & 1 & $0.88 \%$ & 0 & $0.00 \%$ & \\
\hline biclonal (no., \%) & 12 & $4.82 \%$ & 3 & $2.63 \%$ & 9 & $6.67 \%$ & \\
\hline Subtypes of light chains & & & & & & & ${ }^{*} 0.0006$ \\
\hline Kappa & 141 & $59.49 \%$ & 79 & $71.17 \%$ & 62 & $49.21 \%$ & \\
\hline Lambda & 96 & $40.51 \%$ & 32 & $28.83 \%$ & 64 & $50.79 \%$ & \\
\hline (biclonal excluded) & & & & & & & \\
\hline Number of sFLC test (no., \%) & 69 & $27.71 \%$ & 33 & $28.95 \%$ & 36 & $26.67 \%$ & \\
\hline Abnormal sFLC ratio (no., \%) & 12 & $17.39 \%$ & 8 & $24.24 \%$ & 4 & $11.11 \%$ & 0.1506 \\
\hline $\begin{array}{l}\text { Bone marrow plasma cell \% } \\
\text { mean, IQR1-3 (g/dL) }\end{array}$ & 3.3 & $2-5$ & 3.5 & $2-5$ & 3.2 & $2-4$ & 0.65 \\
\hline B2M, median, IQR1-3 (mg/L) & 1.97 & $1.52-2.60$ & 1.52 & $1.45-2.11$ & 2.15 & $1.58-3.02$ & 0.0835 \\
\hline ESR, median, IQR1-3 (mm/h) & 11 & $4-35.5$ & 7 & $2.5-30.5$ & 12.5 & $5-42$ & 0.069 \\
\hline LDH, median, IQR1-3 (IU/L) & 183 & $144-243$ & 159 & $142-221$ & 197 & $145-253$ & 0.0806 \\
\hline $\begin{array}{l}\text { Family history of hematologic } \\
\text { cancer (no., \%) }\end{array}$ & 14 & $6.00 \%$ & 5 & $4.71 \%$ & 9 & $7.09 \%$ & 0.4485 \\
\hline
\end{tabular}

$s F L C$ serum free light chain, IQR1-3 first to third interquartile range, $B 2 M$ beta- 2 microglobin, ESR erythrocyte sedimentation rate, $L D H$ lactate dehydrogenase.

${ }^{*} P$ value $<0.05$

disorders. The reasons leading to the diagnosis of MGUS are reflected in the chief complaints at MGUS diagnosis, categorized by organ systems in Table 3.

The size of M protein was relatively small in our study cohort compared with that reported by others and IQR1-3 between 0.001 to $0.43 \mathrm{~g} / \mathrm{dl} ; 172(69 \%)$ patients had small and unquantifiable $M$ protein at diagnosis, i.e., identified on immunofixation only. The median follow-up duration for these 172 patients was 2.9 years. Only 11 (4\%) patients had a serum $M$ protein level $>1.5 \mathrm{gm} / \mathrm{dl}$. The immunoglobulin type was IgG in 69\%, IgA 10\%, IgM 15\% and other $5 \%$ (biclonal gammopathy, $n=12$ and light chain MGUS, $n=1)$. The type of light chain was kappa in $59 \%$ of the patients and lambda in $41 \%$, when biclonal gammopathy was excluded. There were $69(28 \%)$ patients who had serum free light chain (sFLC) test before progression, 33 (29\%) for patients without immune-related conditions and $36(27 \%)$ with immune-related conditions. The number of abnormal sFLC test was 8 (24\%) for patients without immune-related conditions and $4(11 \%)$ for patients with immune-related conditions $(p=0.20)$. A bone marrow examination was performed in $82(33 \%)$ patients; the median bone marrow plasma cell percentage was $2 \%$ (IQR1-3 2-5\%).

Patients without immune-related conditions tended to have a higher $\mathrm{M}$ protein level compared to patients with immune-related conditions $(p=0.057)$. During the follow up of 2.9 years, the $M$ protein resolved in 36 patients. The median time to the resolution of the $M$ protein was 1.5 years, ranging from 0.2 to 8.6 years. The 
Table 2 Immune-related conditions.

\begin{tabular}{|c|c|c|}
\hline Autoimmune disorders & Inflammatory disoders & Infectious disorders \\
\hline Acquired von Willebrand syndrome & Acute ischemic limb syndrome & Acute cholangitis \\
\hline Ankylosing spondylitis & Alcoholic hepatitis & Bronchitis \\
\hline Antiphospholipid syndrome & Angioedema & Clostridium difficile associated colitis \\
\hline Asthma & Autoimmune myositis & CMV viremia \\
\hline Autoimmune encephalitis & C1 esterase inhibitor deficiency & Cystitis \\
\hline Autoimmune hemolytic anemia & Chronic inflammatory demyelinating polyneuropathy & Disseminated coccidiomycosis \\
\hline Autoimmune hepatitis & Eosinophilic esophagitis & Disseminated herpes zoster \\
\hline Autoimmune neutropenia & Glomerulonephritis & Hepatitis \\
\hline Autoimmune pancreatitis & Gout arthritis & Infectious mononucleosis \\
\hline Autoimmune thyroiditis & Graft-versus-host disease & Neutropenic fever \\
\hline Behcet disease & Hyersensitivity reaction & Perianal abscess \\
\hline Celiac disease & Hypereosinophilic syndrome & Peritonitis \\
\hline Crohn's disease & IgA nephropathy & Seasonal influenza \\
\hline Cryoglobulinemia & Mastocytosis & Sinusitis \\
\hline Diabetes type I & Nephrotic syndrome & \\
\hline Erythema nodosum & Pleuritis & \\
\hline Granulomatosis polyangitis & Post-transplant graft rejection & \\
\hline Immune thrombocytopenic purpura & Seborrheic dermatitis & \\
\hline Inflammatory myopathy & Seronegative spondyloarthropathy & \\
\hline Leukocytoclastic vasculitis & Urticarial vasculitis & \\
\hline \multicolumn{3}{|l|}{ Mixed connective tissue disease } \\
\hline \multicolumn{3}{|l|}{ Multiple sclerosis } \\
\hline \multicolumn{3}{|l|}{ Necrobiotic xanthogranuloma } \\
\hline \multicolumn{3}{|l|}{ Necrotizing vasculitis } \\
\hline \multicolumn{3}{|l|}{ Paroxysmal nocturnal hemoglobinuria } \\
\hline \multicolumn{3}{|l|}{ Polyglandular autoimmune syndrome } \\
\hline \multicolumn{3}{|l|}{ Primary sclerosing cholangitis } \\
\hline \multicolumn{3}{|l|}{ Psoriasis } \\
\hline \multicolumn{3}{|l|}{ Pyoderma gangrenosum } \\
\hline \multicolumn{3}{|l|}{ Sarcoidosis } \\
\hline \multicolumn{3}{|l|}{ Scleroderma } \\
\hline \multicolumn{3}{|l|}{ Sjogren's syndrome } \\
\hline Systemic lupus erythematosus & & \\
\hline Ulcerative colitis & & \\
\hline
\end{tabular}

median size of the $M$ protein of these patients was not quantifiable. The $M$ protein was more likely to resolve in patients with immune-related conditions compared to patients without immune-related conditions (RR $1.91,95 \%$ CI $1.02-3.59 ; p=0.03)$. In the 36 patients who had resolution of their initial $M$ protein, the $M$ protein reappeared in two patients, 6 and 9 years later, respectively.

Overall, 16 (6\%) young MGUS patients developed progression (Table 4): 9 developed SMM, four had active multiple myeloma (MM), one had macroglobulinemia, and two had non-Hodgkin lymphoma. There were 36 
Table 3 Chief complaints for evaluations.

\begin{tabular}{|c|c|c|c|c|c|c|c|c|}
\hline Category & $N$ & Percentage & Category & $N$ & Percentage & Category & $N$ & Percentage \\
\hline Neurologic & 62 & $24.9 \%$ & Rheumatologic & 26 & $10.4 \%$ & Constitutional & 8 & $3.2 \%$ \\
\hline Weakness/paresthesia & 42 & $16.9 \%$ & Back pain & 7 & $2.8 \%$ & Fever & 5 & $2.0 \%$ \\
\hline Seizures & 4 & $1.6 \%$ & Myalgia/arthralgia & 17 & $6.8 \%$ & Dyspnea & 2 & $0.8 \%$ \\
\hline Infarctions & 1 & $0.4 \%$ & Others & 2 & $0.8 \%$ & Others & 1 & $0.4 \%$ \\
\hline Dysautonomia & 1 & $0.4 \%$ & & & & & & \\
\hline Dysphagia & 2 & $0.8 \%$ & $\mathrm{Gl}$ & 24 & $9.6 \%$ & Infectious & 7 & $2.8 \%$ \\
\hline Dystonia & 4 & $1.6 \%$ & Abnormal LFTs & 6 & $2.4 \%$ & $\begin{array}{l}\text { Recurrent/disseminated } \\
\text { infections }\end{array}$ & 7 & $2.8 \%$ \\
\hline \multirow[t]{2}{*}{ Others } & 8 & $3.2 \%$ & Abdominal pain & 4 & $1.6 \%$ & & & \\
\hline & & & Diarrhea & 5 & $2.0 \%$ & Cardiac & 5 & $2.0 \%$ \\
\hline Nephrologic & 40 & $16.1 \%$ & Jaundice & 2 & $0.8 \%$ & cardiomyopathy & 5 & $2.0 \%$ \\
\hline Proteinuria & 13 & $5.2 \%$ & Splenomegaly & 5 & $2.0 \%$ & & & \\
\hline AKI/ESRD & 22 & $8.8 \%$ & Others & 2 & $0.8 \%$ & Others & 3 & $1.2 \%$ \\
\hline \multirow[t]{2}{*}{ Hypercalcemia } & 5 & $2.0 \%$ & & & & N/A & 8 & $3.2 \%$ \\
\hline & & & Dermatologic & 21 & $8.4 \%$ & & & \\
\hline Hematologic & 26 & $10.4 \%$ & Urticaria & 8 & $3.2 \%$ & & & \\
\hline Anemia & 5 & $2.0 \%$ & Pyoderma gangrenaosum & 3 & $1.2 \%$ & & & \\
\hline Leukocytosis & 1 & $0.4 \%$ & Urticaria pigmentosa & 1 & $0.4 \%$ & & & \\
\hline Leukopenia & 5 & $2.0 \%$ & Necrobiotic xanthogranuloma & 1 & $0.4 \%$ & & & \\
\hline Thrombocytosis & 1 & $0.4 \%$ & Others & 8 & $3.2 \%$ & & & \\
\hline Thrombocytopenia & 3 & $1.2 \%$ & & & & & & \\
\hline Lymphadenopathy & 2 & $0.8 \%$ & General evaluations & 19 & $7.6 \%$ & & & \\
\hline Pancytopenia & 1 & $0.4 \%$ & General health exams & 7 & $2.8 \%$ & & & \\
\hline $\begin{array}{l}\text { Relapsed } \\
\text { hematologic } \\
\text { maligancy }\end{array}$ & 2 & $0.8 \%$ & Pretransplant evaluation & 12 & $4.8 \%$ & & & \\
\hline Others & 6 & $2.4 \%$ & & & & & & \\
\hline
\end{tabular}

patients who died and three patients who died of myeloma. The cumulative progression rate to SMM, MM or other related disorders at 5 years and 10 years was $6.0 \%$ and $13.8 \%$, respectively (Fig. 1). In the Cox univariate analysis, the size of $\mathrm{M}$ protein was a significant risk factor for progression (HR 4.23, 95\% CI 2.17-7.91; $p<$ $0.0001)$. Adjusted for the presence of immune-related conditions, the size of $\mathrm{M}$ protein remained a strong risk factor for progression (HR 3.90, 95\% CI 2.0-7.34; $p=$ 0.0002). Age, type of immunoglobulin, type of light chain, and the presence of immune-related conditions did not show statistically significant impact on prognosis in the Cox model.

In Fig. 2, the cumulative risk of progression at 5 years and 10 years for patients with immune-related conditions concurrently present when MGUS was first diagnosed was $1.5 \%$ and $10.1 \%$ respectively; the corresponding rate in patients without immune-related conditions at the time of diagnosis tend to be higher at $12.3 \%$ and $18.9 \%$, respectively (HR 2.36, 95\% CI 0.85-6.52, $p=0.088$ ).

Similar results were seen when the patients in whom the $M$ protein resolved $(n=36)$ were excluded. The cumulative risk of progression at 5 years and 10 years for the entire cohort was $7.6 \%$ and $17.7 \%$, for patients with immune-related conditions was $2.2 \%$ and $14.1 \%$ and for patients without immune-related conditions was $14.0 \%$ and $21.8 \%$ (Log rank $P=0.084$, Wilcoxon $p=0.032)$, (HR 2.49, 95\% CI 0.85-7.30).

\section{Discussion}

Our study explored the prognosis of MGUS in 249 patients younger than 40 years of age. We showed that 
Table 4 Follow-up of the patients.

\begin{tabular}{|c|c|c|c|c|c|c|c|}
\hline Characteristics & $\begin{array}{l}\text { Entire cohort } \\
(N=249)\end{array}$ & & $\begin{array}{l}\text { Patients without immune- } \\
\text { related conditions } \\
(N=114)\end{array}$ & & $\begin{array}{l}\text { Patients with immune- } \\
\text { related conditions } \\
(N=135)\end{array}$ & & $P$ value \\
\hline Follow-up & 2.9 & $0-18.8$ & 1.8 & $0-18.8$ & 4.2 & $0-18.6$ & ${ }^{*} 0.0332$ \\
\hline \multicolumn{8}{|l|}{ Median, range (yr) } \\
\hline \multicolumn{8}{|l|}{ Endpoint (no.) } \\
\hline Any progression & 16 & & 10 & & 6 & & \\
\hline Smoldering multiple myeloma & 9 & & 7 & & 2 & & \\
\hline Multiple Myeloma & 4 & & 2 & & 2 & & \\
\hline Waldenstrom Macroglobulinemia & 1 & & 1 & & 0 & & \\
\hline Non-Hodgkin's lymphoma & 2 & & 0 & & 2 & & \\
\hline Mortality & 36 & & 11 & & 25 & & \\
\hline Resolution of M protein & 36 & $30.51 \%$ & 10 & $20.00 \%$ & 26 & $38.24 \%$ & ${ }^{*} 0.0335$ \\
\hline (No., \%) & & & & & & & \\
\hline
\end{tabular}

${ }^{*} P$ value $<0.05$

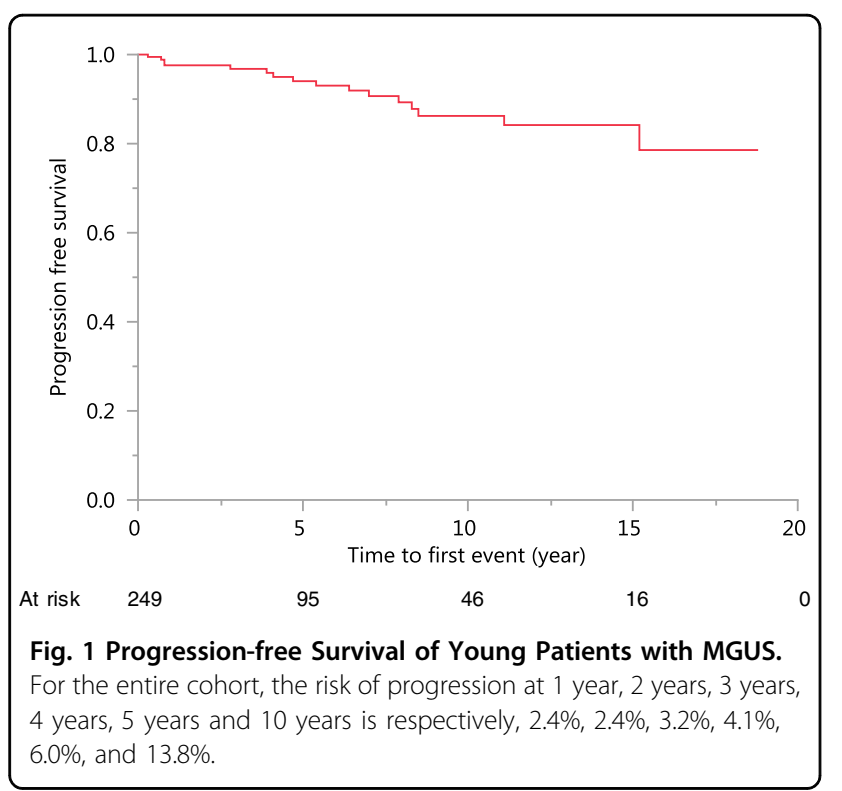

young patients with MGUS had an average progression rate of $1.4 \%$ per year, similar to that of older patients ${ }^{1,2}$. It is uncommon to acquire MGUS at a young age but the early onset of MGUS did not imply a more aggressive or indolent disease process. The risk of progression to plasma cell or lymphoid disorders in young patients was predicted by the size of the serum $M$ protein, a potent risk factor with a 3 fold higher risk of progression per unit increase. This is consistent with the previous studies in MGUS including the older patients where the size of $M$

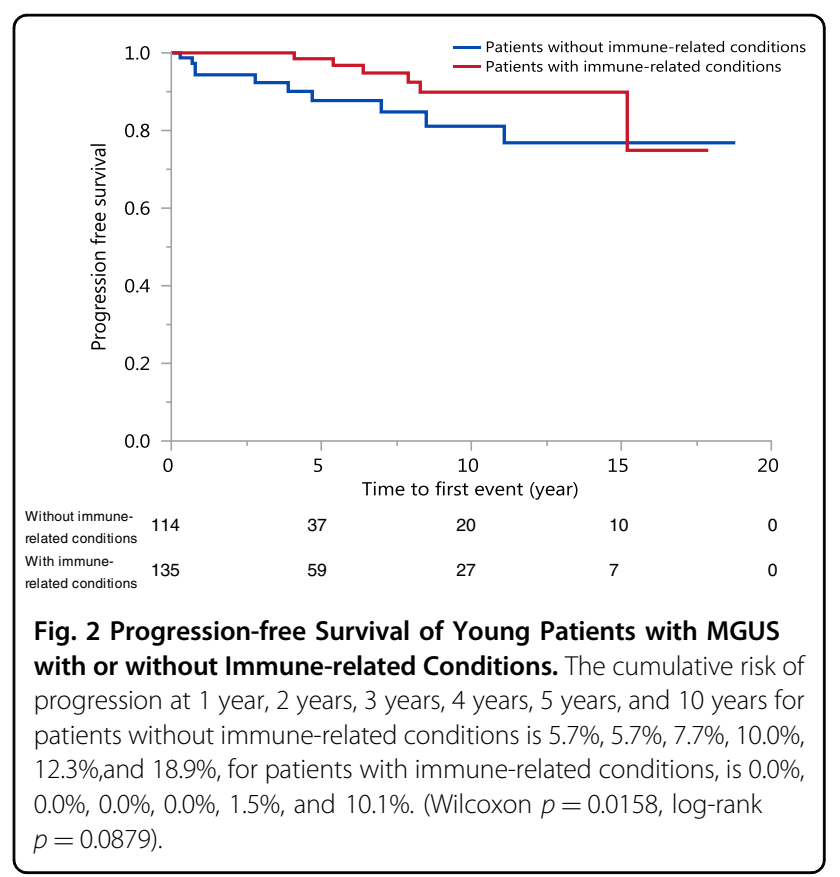

protein was a strong risk factor for progression ${ }^{1}$. The prognostic impact of the size of $\mathrm{M}$ protein is also evident among young MGUS patients in our study.

Our study did not find a statistically significant difference in the risk of progression between patients with immune-related conditions and patients without immune-related conditions, although there was a trend towards higher risk of progression in those without a coexisting immune disorder (HR 2.36, 95\% CI 0.85-6.52, 
$p=0.088)$. In the early phase of follow-up, there was a higher rate of progression in patients without immunerelated conditions than in the patients with immunerelated conditions. In the overall long-term follow-up, this difference in progression rate disappeared.

This finding could be explained by the lead-time bias $^{12,13}$; the survival time was artificially prolonged by early diagnosis of disease. In our study, it is likely that the patients with immune-related conditions were diagnosed earlier in the disease course of MGUS than those without immune-related conditions. They were diagnosed during the tests performed as part of clinical care for the autoimmune condition. It is possible that young MGUS patients without immune-related conditions may have acquired the potential of developing plasma cell disorder in the past ${ }^{10,14-16}$. In the early follow-up phase of our study, those patients without immune-related conditions had a higher rate of progression to plasma cell proliferative disorders because they had a longer history of MGUS compared with the patients with immune-related conditions who has a shorter history of MGUS. In the long-term follow up as shown in our study, there is no difference in progression free survival between the two groups of the patients.

The size of $M$ protein in our study is relatively small compared to those in previous studies ${ }^{2,5}$. There are two potential explanations for this observation. First, our young MGUS patients were likely diagnosed in an earlier stage of their disease process rather than the older patients who had a longer history of undiagnosed MGUS until it is identified incidentally. The size of $M$ protein gradually increases in the majority of patients over the disease course ${ }^{17}$. It is possible that the immune-related conditions is the early trigger for development of plasma cell disorders ${ }^{14}$, as the size of $\mathrm{M}$ protein was frequently reported as unquantifiable in the setting of immunerelated conditions ${ }^{18-20}$.

Second, the unquantifiable $M$ protein in patients with immune-related conditions might not represent bona-fide MGUS as some of them did not persist ${ }^{21}$. There were 36 patients in whom we observed the disappearance of $M$ protein. In a smaller number of patients, we could not ascertain the status of the $M$ protein as these patients were lost to follow-up after the diagnosis of MGUS. We find that the $M$ protein was more likely to resolve in the patients with immune-related conditions compared with the patients without immune-related conditions. The patients with immune-related conditions also had a lower serum concentration of $\mathrm{M}$ protein. The pathophysiology behind the resolution of $\mathrm{M}$ protein is not clear. Murray et al reported that unquantifiable $M$ proteins were less likely to persist as $30 \%$ of the unquantifiable $M$ proteins disappeared in their study. They also stated among the patients with non-persistent $\mathrm{M}$ proteins, $55 \%$ of them had immunologic disease or received immune-modulating medication $^{21}$. A few case series revealed similar findings that the presence of $M$ protein was of small size and transient during acute renal failure and various infectious diseases $^{18-20,22,23}$. The trend that patients without immune-related conditions may have higher rate of progression would be explained by the hypothesis that unquantifiable $M$ protein does not represent the true MGUS with potential to progress. The persistence of $M$ protein could also be interrupted by immunosuppressive medications including steroids which may reduce the serum $\mathrm{M}$ protein as well as cyclophosphamide which has anti-myeloma activity ${ }^{24-26}$.

The long-term prognosis of transient paraproteinemia is worth investigation ${ }^{27}$. Even though the $M$ protein disappeared, it could recur. In the 36 patients of whom the M protein resolved, two patients were observed with the recurrence of the $M$ protein after 6 and 9 years, respectively from its initial resolution. The type of the recurrent $M$ protein was the same as the initial one in these two patients. Both patients were chronically on immunosuppression for their underlying diseases and the recurrence was not associated with the discontinuation of their immunosuppression. This finding is contrary to the idea that the transient paraproteinemia is a benign phenomenon. Long-term follow-up is needed to better understand this phenomenon.

There are limitations to the current study. We were not able to assess the prognostic effect of the serum free light chain ratio in our young MGUS population due to the small number of patients with available results. It is possible that the serum free light chain ratio plays a major role in prognosis as in older population but needs further study. The exact contribution of steroids to the resolution of the M protein is not known in our study as we did not have the percentage of the patients who were on steroids. It is known that steroids are one of the myeloma treatments could reduce the $M$ protein. The follow-up duration is short in our study. Many patients who came to Mayo Clinic for complex diseases and were incidentally found to have MGUS at a young age. They lost follow up after several visits at Mayo Clinic. Possibly, their MGUS was followed up by their primary care doctor. SMM can have very protracted asymptomatic course that does not require treatment. It will provide more clinical value if future researches can include if patients require chemotherapy after they progress to SMM.

\section{Conclusions}

Young patients with MGUS have a similar risk of progression as in older patients, $1.4 \%$ per year. Approximately $50 \%$ are diagnosed in the setting of immune-related disorders. When occurring in the setting of immune-related disorders, the $M$ protein is 
smaller, more likely to resolve, and may have a lower risk of progression than in patients in whom MGUS is detected without concurrent immune-related disorder. The current study adds to our understanding of monoclonal gammopathies and their etiology.

\section{Author contributions}

L.P. designed the study, collected the data, performed the analysis, and wrote the manuscript. S.V.R designed the study, performed the analysis, revised the manuscript critically, and approved the final version of the manuscript. P.K., F.B., A.D., M.G., M.L., R.K. performed patients' management, revised the manuscript critically and approved the final version of the manuscript. S.K.K. designed the study, performed the analysis and wrote the manuscript and approved the final version of the manuscript.

\section{Conflict of interest}

The authors declare that they have no conflict of interest.

\section{Publisher's note}

Springer Nature remains neutral with regard to jurisdictional claims in published maps and institutional affiliations.

Received: 17 May 2020 Accepted: 28 October 2020

Published online: 01 February 2021

\section{References}

1. Kyle, R. A. et al. A long-term study of prognosis in monoclonal gammopathy of undetermined significance. N. Engl. J. Med. 346, 564-569 (2002).

2. Kyle, R. A. et al. Long-term follow-up of monoclonal gammopathy of undetermined significance. N. Engl. J. Med. 378, 241-249 (2018).

3. Landgren, $\mathrm{O}$. et al. Prevalence of myeloma precursor state monoclonal gammopathy of undetermined significance in 12372 individuals 10-49 years old: a population-based study from the National Health and Nutrition Examination Survey. Blood Cancer J. 7, e618 (2017).

4. Kyle, R. A. et al. Prevalence of monoclonal gammopathy of undetermined significance. N. Engl. J. Med. 354, 1362-1369 (2006).

5. Lindqvist, E. K. et al. Personal and family history of immune-related conditions increase the risk of plasma cell disorders: a population-based study. Blood 118, 6284-6291 (2011).

6. Brown, L. M., Gridley, G., Check, D. \& Landgren, O. Risk of multiple myeloma and monoclonal gammopathy of undetermined significance among white and black male United States veterans with prior autoimmune, infectious, inflammatory, and allergic disorders. Blood 111, 3388-3394 (2008).

7. McShane, C. M. et al. Prior autoimmune disease and risk of monoclonal gammopathy of undetermined significance and multiple myeloma: a systematic review. Cancer Epidemiol., Biomarkers Prevent. 23, 332-342 (2014).

8. Hemminki, K. et al. Effect of autoimmune diseases on incidence and survival in subsequent multiple myeloma. J. Hematol. Oncol. 5, 59-59 (2012).
9. Choi, J. W., Han, S. W., Kwon, K. T. \& Kim, G. W. Early onset multiple myeloma in a patient with systemic lupus erythematosus: a case report and literature review. Clin. Rheumatol. 29, 1323-1326 (2010).

10. Morgan, G. J., Walker, B. A. \& Davies, F. E. The genetic architecture of multiple myeloma. Nat. Rev. Cancer 12, 335 (2012).

11. Rasmussen, $\mathrm{T}$. et al. Identification of translocation products but not K-RAS mutations in memory B cells from patients with multiple myeloma. Haematologica 95, 1730-1737 (2010).

12. Nair, H. S., Knight, S. R., McKenzie, C., MacDonald, A. J. \& Macdonald, A. Age at death and the effect of lead-time bias in patients with colorectal cancer: a 10-year follow-up. Colorectal Dis. 21, 775-781 https://doi.org/ 10.1111/codi.14602 (2019).

13. Hutchison, G. B. \& Shapiro, S. Lead time gained by diagnostic screening for breast cancer. J. Natl Cancer Inst. 41, 665-681 (1968).

14. Shimanovsky, A., Alvarez Argote, J., Murali, S. \& Dasanu, C. A. Autoimmune manifestations in patients with multiple myeloma and monoclonal gammopathy of undetermined significance. BBA Clin. 6, 12-18 (2016).

15. Walker, B. A. et al. Characterization of IGH locus breakpoints in multiple myeloma indicates a subset of translocations appear to occur in pregerminal center B cells. Blood 121, 3413-3419 (2013).

16. Walker, B. A. et al. Intraclonal heterogeneity is a critical early event in the development of myeloma and precedes the development of clinical symptoms. Leukemia 28, 384-390 (2014).

17. Landgren, O. et al. Monoclonal gammopathy of undetermined significance (MGUS) consistently precedes multiple myeloma: a prospective study. Blood 113, 5412-5417 (2009).

18. Widmer, C. C., Balabanov, S., Schanz, U. \& Theocharides, A. P. A. Transient paraproteinemia after allogeneic hematopoietic stem cell transplantation is an underexplored phenomenon associated with graft versus host disease. Oncotarget 8, 106333-106341 (2017).

19. Enko, D., Emhofer, J., Farid, G. \& Kriegshauser, G. Transient monoclonal gammopathy in a 2-year-old child with combined viral and bacterial infection. Clin. Chem. Lab. Med. 56, e310-e312 (2018)

20. Conant, J. L., Rabinowitz, I. \& Zhang, Q. Y. Transient monoclonal gammopathy induced by Candida fungemia. Hum. Pathol. 75, 154-158 (2018).

21. Murray, D. L. et al. Laboratory persistence and clinical progression of small monoclonal abnormalities. Am. J. Clin. Pathol. 138, 609-613 (2012).

22. Yang, $Y$. et al. Monoclonal gammopathy in rheumatic diseases. Clin. Rheumatol. 37, 1751-1762 (2018).

23. Presle, A. et al. An acute monoclonal gammopathy? Ann. Biol. Clin. 73 185-189 (2015).

24. Mass, R. E. A comparison of the effect of prednisone and a placebo in the treatment of multiple myeloma. Cancer Chemother. Rep. 16, 257-259 (1962).

25. Burwick, N. \& Sharma, S. Glucocorticoids in multiple myeloma: past, present, and future. Ann. Hematol. 98, 19-28 (2019).

26. Sharma, S. \& Lichtenstein, A. Dexamethasone-induced apoptotic mechanisms in myeloma cells investigated by analysis of mutant glucocorticoid receptors. Blood 112, 1338-1345 (2008).

27. Gonzalez Garcia, M. E. et al. A series of 618 cases of monoclonal gammopathies of undetermined significance: predictive factors of disappearance of monoclonal component or evolution to malignant gammopathies. Rev. Clin. Espanola 208, 288-294 (2008). 